\title{
Rapid Mechanical Property Tomograms Generated by Indentation Testing
}

K. E. Johanns ${ }^{1}$, B. Crawford ${ }^{1}$, and K. H. Parks ${ }^{1}$

1. Nanomechanics, Inc., Oak Ridge, USA.

Instrumented indentation testing, or nanoindentation, is a mechanical testing technique that can locally measure the elastic modulus and hardness of a material at nano- and micro-scales. Over the past 2 decades, nanoindentation has added the capability to measure mechanical properties continuously as a function of indenter penetration depth, greatly enhancing the characterizatipon of thin films, coatings, multi-layer materials, and surface gradients. Here, we show how the depth sensing indentation technique can be extended to creating mechanical property tomograms in a reduced period of time. Critical assumptions, limitations, and unique outcomes of the technique are discussed along with examples on different material systems (e.g., a multi-layer chip, a two-phase alloy, and a patterned surface). An example of a nanoindentation mechanical property tomogram is shown in Fig. 1.

A nanoindenter makes four critical measurements: (1) The applied load on the sample, $P$; The indenter depth of penetration, $h$; (3) The elastic contact stiffness, $S$; and (4) The contact area, $A$ [1]. Material properties can then be assigned at different spatial locations (e.g., x, y, and h) by performing an array of indents. However, this process has often taken a significant amount of time to create a mechanical property tomogram of sufficient resolution. In order to speed up the indentation process, the following critical aspects of ultra-fast instrumented indentation testing need to be addressed:

- Dynamic characterization of equipment (mass, damping, and stiffness)

- Controlled single degree of freedom actuation

- Accurate and fast sensors with high data acquisition rates

A multilayer thin film consisting of porous silica and metallization layers was chosen here to highlight the results of the mechanical property tomogram technique (Fig. 2). The sample was chosen because of the large differences in mechanical behavior as a function of position. An iNano Nanoindenter (Nanomechanics, Inc.) equipped with a diamond Berkovich indenter was used to indent the multilayer material. A $30 \times 30$ array of indents with a $1.5 \mathrm{um}$ spacing were made to a depth of $200 \mathrm{~nm}$ at an indentation strain rate of $3.0 \mathrm{~s}^{-1}$. The total testing time was $\sim 60$ minutes.

The results of the indentation testing showed significant differences in the mechanical behavior of the multilayer thin film. The technique was able to distinguish between the different metallization layers as well as capture interfaces between the different components as seen by the sharp contrast in data in Fig. 3. Mechanical property tomograms offer the ability to examine the mechanical response (e.g., adhesion, fracture, stiffness, and deformation resistance) in complicated, small-scale parts where more traditional techniques are limited [2]. 


\section{References:}

[1] W. C. Oliver and G. M. Pharr, JMR, 1992.

[2] The authors would like to thank T. Shaw and H. Huang of the TJ Watson Research Center of IBM and the Albany Nanotechnology Research Center of IBM, respectively, for the multilayer thin film sample.

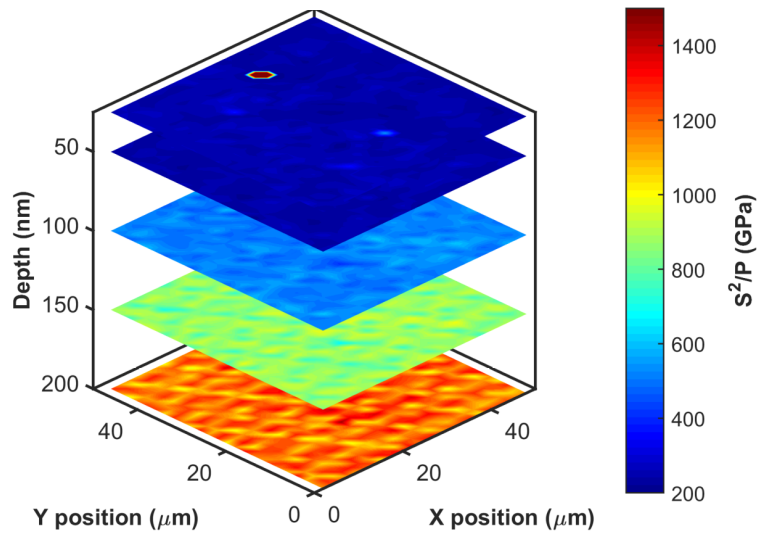

Figure 1. Example nanoindentation mechanical property tomogram.

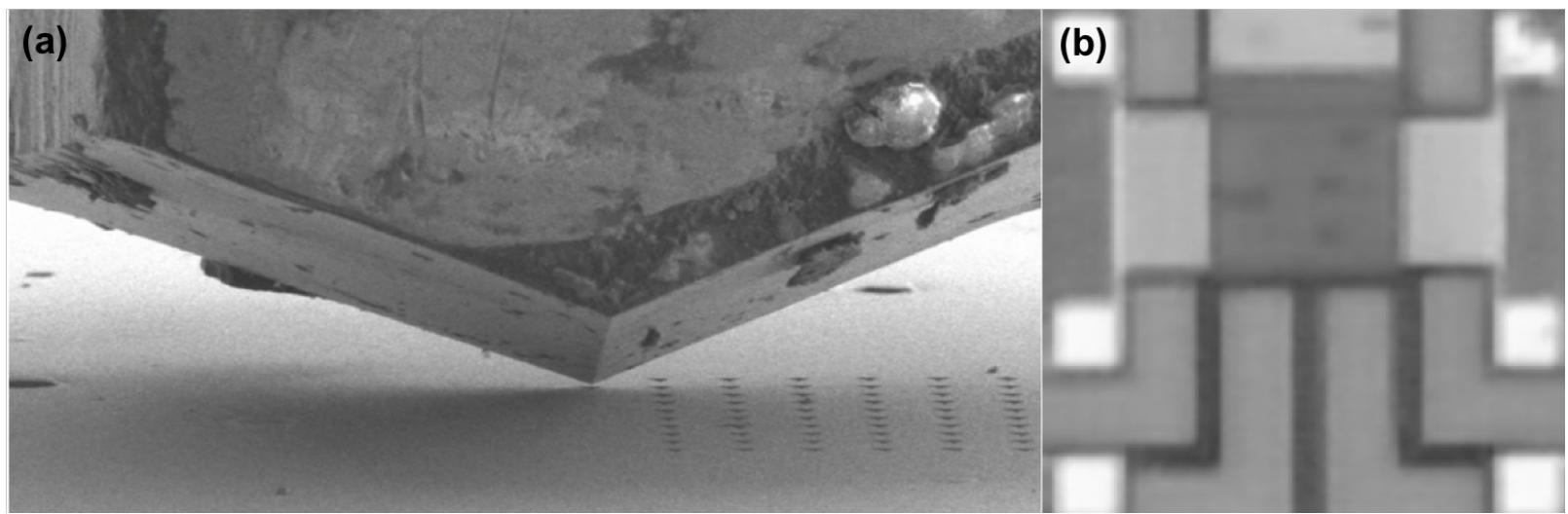

Figure 2. Multilayer thin film: (a) Scanning electron microscope image; and (b) light optical microscope image.
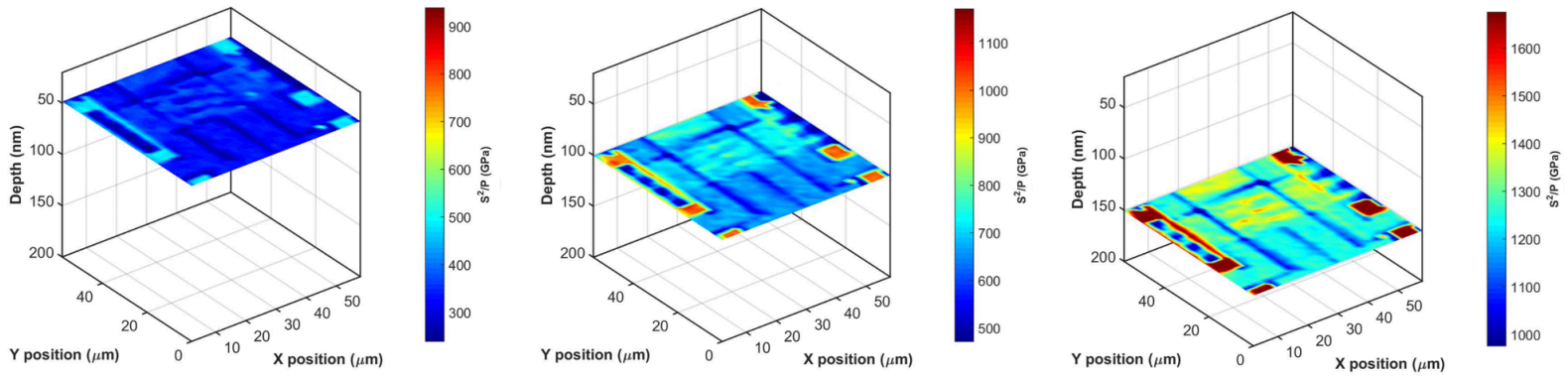

Figure 3. Mechanical property tomogram slices of a multilayer thin film at indentation depths of 50nm, $100 \mathrm{~nm}$, and $150 \mathrm{~nm}$. 